was buried while the decomposition of the hind limbs was still at an early stage . $^{8}$

Of special interest is the clearly defined contour of the soft body. Because of the slight rotation, the outlines of the left and right sides of the carcass differ markedly. On the left side, the dense and rather long-haired pelage extends from the muzzle to the rump; long vibrissae and parts of the external ear are also visible. The outline of the right side, however, is formed by a thin, sparsely haired, and laterally expanded membrane which is spread out between the neck, wrist, ankle and proximal tail vertebrae. The boundary between the membranous skin and the compact, densely haired body is particularly obvious in front of the foreleg. The outline along the trunk is straight and parallel to the long axis of the body. We conclude that the membrane acted as a patagium and Eomys quercyi was a glider (Fig. 4). Its preservation in a dorsoventral position results obviously from the presence of the gliding membranes. This discovery contradicts the supposition, based on the distribution and abundance of extant gliders, that the evolution of gliding and the occupation of the gliding niche were linked to rich tropical-forest ecosystems and that particular tropical vegetation structures figured prominently in this connection ${ }^{9,10}$.

Glissant locomotion has developed by parallel evolution in four rodent families. Among extant rodents, these are flying squirrels and scaly-tailed flying squirrels. The patagia are supported in many forms by cartilaginous struts or calcars, which originate from the carpus in sciurids and from the olecranon process in anomalurids. Presumably, Eomys quercyi, like anomalurids, had a long strut from the elbow; a rod extends from the right olecranon of the Enspel specimen and seems to represent the medially displaced calcar. Flying squirrels are characterized by the elongation of the distal elements of the forelimbs (ratio of radius : humerus $=0.99$ 1.13 (ref. 11), which is not found in either Anomalurus (0.90) or E. quercyi $(0.85)$. The dormouse family also includes a glider: its living members are non-gliding, mainly arboreal animals, the sole glissant species being Glirulus aff. lissiensis Hugueney \& Mein, 1965 from the late Miocene of the Ardèche, France ${ }^{12}$. The fossil dormouse is similar to E. quercyi from Enspel in the dorsoventral position of the carcass and the imprint of the patagium. Note that it is referred by dental characters to the same genus as the living non-gliding Japanese species, Glirulus japonicus (Schinz, 1845).

Accordingly, we cannot infer gliding adaptations for all eomyids, or even all species of the genus Eomys, from this specimen of $E$. quercyi. Eomys is much too frequent and diverse in many European localities for one to assume that all species were gliders. Previous postcranial evidence of eomyids consisted of parts of a humerus, pelvis, femur, tibia and calcaneum from the North American middle Oligocene ${ }^{13}$, and suggested a cursorial locomotion as well as 'an aberrant adaptation' ${ }^{14}$. The highly specialized locomotor strategy of $E$. quercyi, however, could not have been inferred from the living members of the clade (heteromyids and geomyids) or from previously available fossils of the family Eomyidae.

Received 25 July; accepted 13 November 1995.

1. Engesser, B. Schweiz. Paläont. Abh. 112, 1-144 (1990).

2. Korth, W. W. The Tertiary Record of Rodents in North America (Plenum, New York, 1994).

3. Qiu, Zh.-d. Natn. Sci. Mus. Monogr., Tokyo 8, 49-55 (1994).

3. Qiu, Zh.-d. Natn. Sci. Mus. Monogr., Tokyo 8, 49-55 (1994).
4. Fahlbusch, V. in Evolutionary Relationships among Rodents. A Multidisciplinary Analysis (ed.

Luckett, P. W. \& Hartenberger, J.-L.) 617-629 (Plenum, New York, 1985).

5. Alvarez Sierra, M. A. Scripta geol. 86, 1-207 (1987).

6. Comte, B. \& Vianey-Liaud, M. C.r. Acad. Sci, Paris 304, 951-954 (1987).

7. Burbank, D. W. et al. Eclogae geol. Helv. 85, 399-431 (1992).

8. Wuttke, W. in Messel. An Insight into the History of Life and of the Earth (ed. Schaal, S. \& Ziegler, W.) 263-275 (Clarendon, Oxford, 1992)

9. Emmons, L. H. \& Gentry, A. H. Am. Nat. 121, 513-524 (1983).

10. Dudley, R. \& DeVries, P. Biotropica 22, $432-434$ (1990).

11. Thorington, R. W. \& Heaney, L. R. J. Mamm. 62, 101-114 (1981).

12. Mein, P. \& Romaggi, J.-P. Geobios 13, 45-50 (1991)

13. Cope, E. D. Rep. U.S. geol. Surv. Terr. 3, 1-1009 (1884).

14. Wood A. E. Trans Am phil. Soc. 28, 155-269 (1937).

15. Koenigswald, W. von, Storch, G. \& Richter, G. in Messel. An Insight into the Histon of Life and of the Earth (ed. Schaal, S. \& Ziegler, W.) 217-222 (Clarendon, Oxford, 1992).

16. Mai, D. H. \& Walther, H. Abh. staatl. Mus. Mineral. Geol., Dresden 38, 1-230 (1991).

ACKNOWLEDGEMENTS. We thank Jin Meng and J. J. Hooker for comments. P. Schäfers skillfully prepared the specimen described herein.

\section{A canine distemper virus epidemic in Serengeti lions (Panthera leo)}

\author{
Melody E. Roelke-Parker* $\dagger$, Linda Munson $¥ \#$, \\ Craig Packer*\$, Richard Kock\|, \\ Sarah Cleaveland* \#, Margaret Carpenter ${ }^{\text {is }}$, \\ Stephen J. O'Brien ${ }^{\text {ts }}$, Andreas Pospischil**, \\ Regina Hofmann-Lehmann $\dagger \dagger$, Hans Lutz $\dagger \dagger$, \\ George L. M. Mwamengele $\ddagger \ddagger$, M. N. Mgasa $\ddagger \ddagger$ \\ G. A. Machange $\S$, Brian A. Summers \|\| \\ \& Max J. G. AppelTी
}

* Serengeti Wildlife Research Institute, Tanzania National Parks, Arusha, Tanzania

† Messerli Foundation, Zurich, Switzerland

$\ddagger$ Department of Pathology, College of Veterinary Medicine, University of Tennessee, Knoxville, Tennessee 37901, USA

$\S$ Department of Ecology, Evolution, and Behavior, University of Minnesota, St Paul, Minnesota 55108, USA

$\|$ Kenya Wildlife Service, Nairobi, Kenya

I Institute of Zoology, London NW1 4RY, UK

\# London School of Hygiene and Tropical Medicine, London WC1E 7HT, UK

Laboratory of Viral Carcinogenesis, National Cancer Institute, Frederick, Maryland 21702, USA

** Institute of Veterinary Pathology, and †† Department of Veterinary Internal Medicine, University of Zurich, 8057 Zurich, Switzerland $\ddagger \ddagger$ Department of Veterinary Pathology, Sokoine University of Agriculture, Morogoro, Tanzania

$\S \S$ Veterinary Investigation Center, Arusha, Tanzania

II || Department of Pathology, and Tा James Baker Institute of Animal Health, College of Veterinary Medicine, Cornell University, Ithaca, New York 14853, USA

CANINE distemper virus (CDV) is thought to have caused several fatal epidemics in canids within the Serengeti-Mara ecosystem of East Africa, affecting silver-backed jackals (Canis mesomelas) and bat-eared foxes (Otocyon megalotis) in 1978 (ref. 1), and African wild dogs (Lycaon pictus) in 1991 (refs 2, 3). The large, closely monitored Serengeti lion population ${ }^{4,5}$ was not affected in these epidemics. However, an epidemic caused by a morbillivirus closely related to CDV emerged abruptly in the lion population of the Serengeti National Park, Tanzania, in early 1994, resulting in fatal neurological disease characterized by grand mal seizures and myoclonus; the lions that died had encephalitis and pneumonia. Here we report the identification of CDV from these lions, and the close phylogenetic relationship between $\mathrm{CDV}$ isolates from lions and domestic dogs. By August 1994, 85\% of the Serengeti lion population had anti-CDV antibodies, and the epidemic spread north to lions in the Maasai Mara National reserve, Kenya, and uncounted hyaenas, bat-eared foxes, and leopards were also affected.

In early 1994, six lions in the Serengeti National Park, Tanzania were observed with grand mal seizures, and three other lions developed facial and forelimb myoclonus (recurrent twitching). Additional lions were noted to be disoriented, ataxic and profoundly depressed. Between January and March 1994, 11 lion carcasses were found, representing a dramatic increase in mortality from previous years and indicating that a serious epidemic was emerging.

To investigate the epidemic, tissue and serum samples were obtained from 23 lions that died or were killed in a moribund state, 13 live lions with obvious signs of disease, and 72 apparently healthy, anaesthetized lions. Sera from 111 healthy lions sampled between 1984 and 1994 (refs 6-8) were also analysed to track previous exposure of this population to viruses. Tissues from 19 


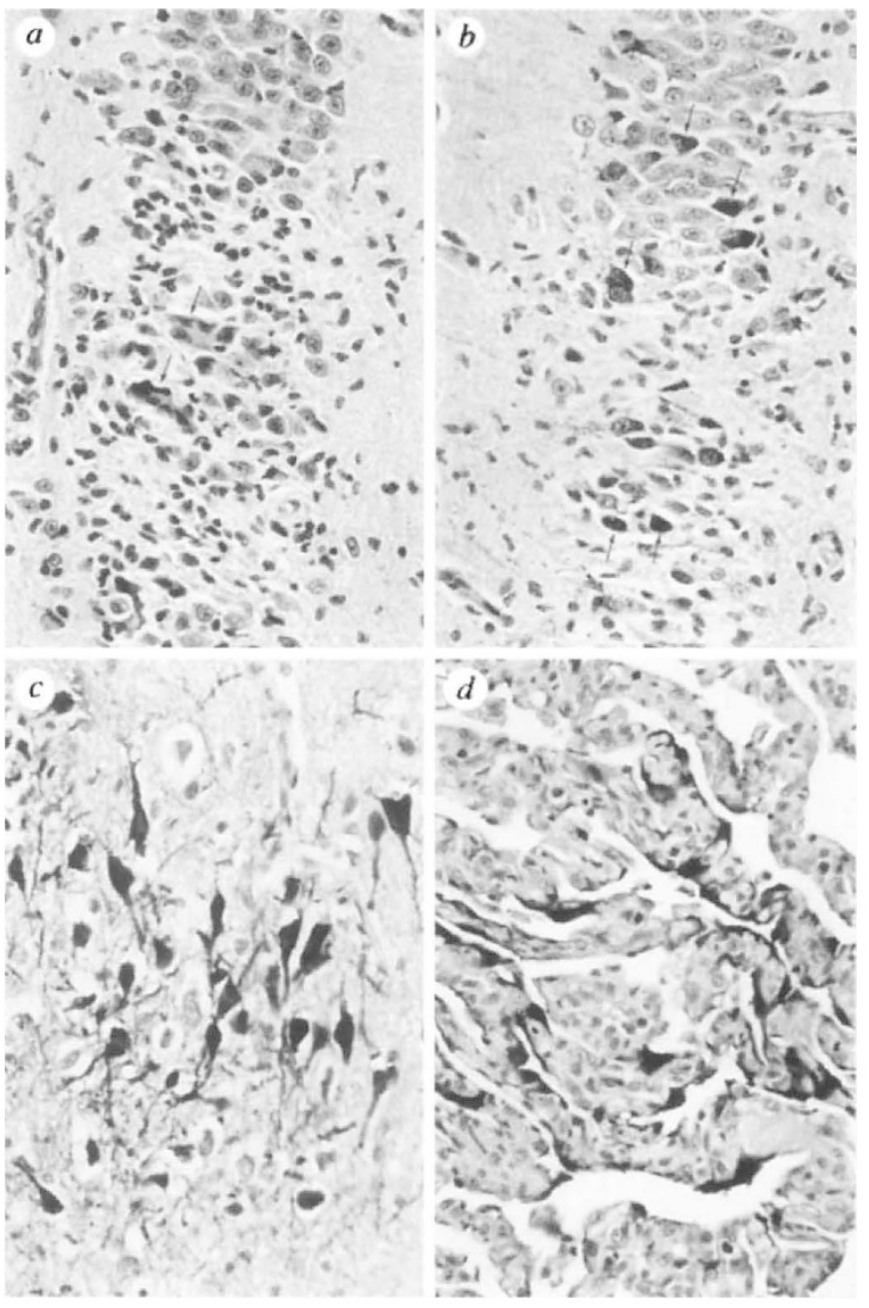

dead lions, examined by histopathology, had either encephalitis, interstitial pneumonia, and/or lymphocytic depletion in lymph nodes and spleen (Fig. 1; Table 1). Rare multinucleated syncytia and intranuclear and/or intracytoplasmic viral inclusions characteristic of morbilliviral infection ${ }^{9}$ were also found in these lions (Table 1). Because these lesions were seen in zoo cats in the United States in the 1991 and 1992 canine distemper epidemics ${ }^{10}$, we used monoclonal ${ }^{11}$ and polyclonal CDV antibodies to confirm that CDV nucleocapsid antigens were present in affected tissues. We then tested all available lion sera for neutralizing antibody titres to $\mathrm{CDV}^{10,12}$, and found that 63 of the 72 apparently healthy lions and 8 of the 11 sick lions sampled in 1994 had CDV titres (Fig. 2a). We isolated CDV from the cerebrospinal fluid of one lion cub with grand mal seizures that subsequently died with CDV encephalitis ${ }^{13}$. The monoclonal antibody-binding pattern of this virus was compared to binding patterns of viruses isolated from: a bat-eared fox (O. megalotus), a spotted hyaena (Crocuta crocuta), and a domestic dog that died during the 1994 Serengeti epidemic; with CDV isolated from a lion during the 1992 California epidemic; with CDV isolated from a raccoon (Procyon lotor) and fox (Vulpes vulpes); and with a virulent CDV (A75-17, 1975) and two strains of attenuated CDV from domestic dogs (Rockborn 1958 and Onderstepoort 1948). Monoclonal antibodies were donated by C. Orvell (Huddinge, Sweden), and were directed against viral nucleoprotein $(\mathrm{N})$, polymerase $(\mathrm{P})$, fusion glycoprotein $(\mathrm{F})$, and haemagglutinin glycoprotein $(\mathrm{H})$. Lymphocytes infected with the Serengeti lion virus bound the same group of CDV monoclonal antibodies as cells infected with these other viruses, suggesting that the lion morbillivirus was CDV. To characterize further the lion morbillivirus, the genomic sequences of the CDV P gene, a conserved region of the virus, were amplified from buffy-coat
FIG. 1 Characteristic histopathological changes and intralesional immunoreactive viral nucleocapsid antigens in tissues of African lions infected with canine distemper virus. a, Haematoxylin- and eosin-stained section of the brain from a lion with CDV encephalitis $(\times 350)$. The dentate gyrus in the hippocampus has neuronal loss, dense gliosis and occasional multinucleated giant cells (arrows). $b$, Immunohistochemical identification by monoclonal antibody N3.991 against CDV-nucleocapsid protein in a similar area to a. Several neurons contain CDV antigen in the cytoplasm (some indicated by arrows) ( $\times 350)$. c, Immunohistochemical identification of CDV nucleocapsid in a parahippocampal gyrus $(\times 350)$. Many neurons are strongly positive for CDV antigen in perikarya and neurites. $d$, Immunohistochemical identification of CDV nucleocapsid proteins in lung with interstitial pneumonia, characterized by type II pneumocyte hyperplasia and alveolar septal thickening $(\times 350)$.

METHODS. Tissues were fixed in $10 \%$ formalin, embedded in paraffin, sectioned at 5-7 $\mu \mathrm{m}$ and stained with haematoxylin and eosin. Immunohistochemical procedures were applied to sections of formalin-fixed, paraffin-embedded tissues that had typical CDV lesions on stained sections to demonstrate intralesional viral antigens ${ }^{10}$. Paraffin-embedded tissues had the paraffin removed, were treated to remove endogenous peroxidase, then incubated with a mouse monoclonal antibody to a CDV-N protein (MAb N3.991) ${ }^{11}$, or with rabbit primary polyclonal antibody raised with the Rockborn strain of CDV. A commercial avidin-biotin kit was used to identify sites of monoclonal antibody binding to tissues, and a commercial peroxidaseantiperoxidase kit was used for polyclonal antibodies, then tissue sections were counterstained with Gill's haematoxylin, dehydrated and mounted with Permount. Negative controls were duplicate sections stained using a monoclonal antibody for influenza virus replacing CDV monoclonal antibodies, and positive controls were brain sections from a confirmed case of CDV in a domestic dog.

lymphocytes of two lions with neurological signs. Phylogenetic relationships between the P-gene sequences of the two RT-PCRderived lion CDV and the P-gene sequences of other morbilliviruses were then examined. These analyses (Fig. 3) indicated that Serengeti lion CDV virus is closely related to the Onderstepoort strain of canine distemper virus isolated from a domestic dog in South Africa. The statistics of the epidemic are given in Table 1.

Later in 1994, CDV-infected lions were identified in the northern and western areas of the Serengeti National Park and in the Maasai Mara National Reserve in Kenya (Fig. 2b). Of 54 apparently healthy lions sampled from October 1994 to March 1995 in the Mara and neighbouring areas of Kenya, 23 had high serum titres of CDV antibodies, suggesting recent exposure.

During 1994, 39 CDV-associated lion deaths were documented, but this is probably an underestimation of true mortality statistics because most of the Serengeti lion population outside the Seronera study area is not under close observation. The overall lion population in the Serengeti ecosystem, estimated at 3,000 before the outbreak, is now estimated at 2,000. In 1994 and 1995, CDV-related deaths were also confirmed in seven spotted hyaenas $(C$. crocuta) by pathology and virology. Because CDV is historically not pathogenic in lions or hyaenas ${ }^{9,14}$, the emerging biotype of CDV has apparently extended its host range. During the epidemic, CDVinduced disease also was confirmed in two bat-eared foxes, and CDV-like neurological disease was observed in a common jackal (Canis aureus) and two silver-backed jackals, indicating that the Serengeti CDV biotype conserves its pathogenicity for canids.

The magnitude of this epidemic may be explained in part by the lion population being immunologically naive to CDV when it was introduced in 1994. All but one of the 34 lions sampled between 1990 and 1993 were seronegative, and the scropositive lion was 
Fig. 2 a, Temporal patterns of mortality and seroconversion in the longterm study population of lions in the Serengeti National Park. Top, number of lions resident in the long-term study area, measured monthly from August 1974 to February 1995 (refs 4, 5). Middle, prevalence of anticanine distemper virus (CDV) antibodies ${ }^{10}$ in Serengeti lions from 1990 to 1994. The single seropositive lion in 1993 was sampled in December. Bottom, age-specific mortality (proportion dying per year) in female lions in the study population. The solid line represents the combined mortality rate from 1966 to 1993; the broken line represents mortality in 1994. Male mortality was comparable to female mortality, but was not included here because male disappearances could also be caused by normal, nondisease-related dispersals $s^{4,5} . b$, Chronology and geographic distribution of CDV disease in the Serengeti ecosystem. The months in boxes are dates in 1994 when lions were first observed with neurological disease. The proportions of CDV-seropositive domestic dogs in regions surrounding the Serengeti National Park are represented by pie charts for 1992 to 1994 ( $n$, number of dogs tested). The domestic dog that was confirmed to die from canine distemper disease in September 1994 was located in the Ngorongoro Conservation Area. Endemic infection was confirmed in the Serengeti district where CDV seropositivity was detected in pups $(<12$ months old) in each year of the study (1992-1994). This district also has one of the highest dog population densities. In the Loliondo and Ngorongoro areas, seropositivity was detected in pups in 1994 (3/7), but not in 1992 $(0 / 6)$ or $1993(0 / 17)$.

METHODS. Sera were tested against the Onderstepoort strain of CDV adapted to Vero cells as described previously ${ }^{12}$. Sera were also tested against CDV isolated from a lion during the California epidemic (A92-27/ 20). Log titres of 1.0 or greater were considered positive.
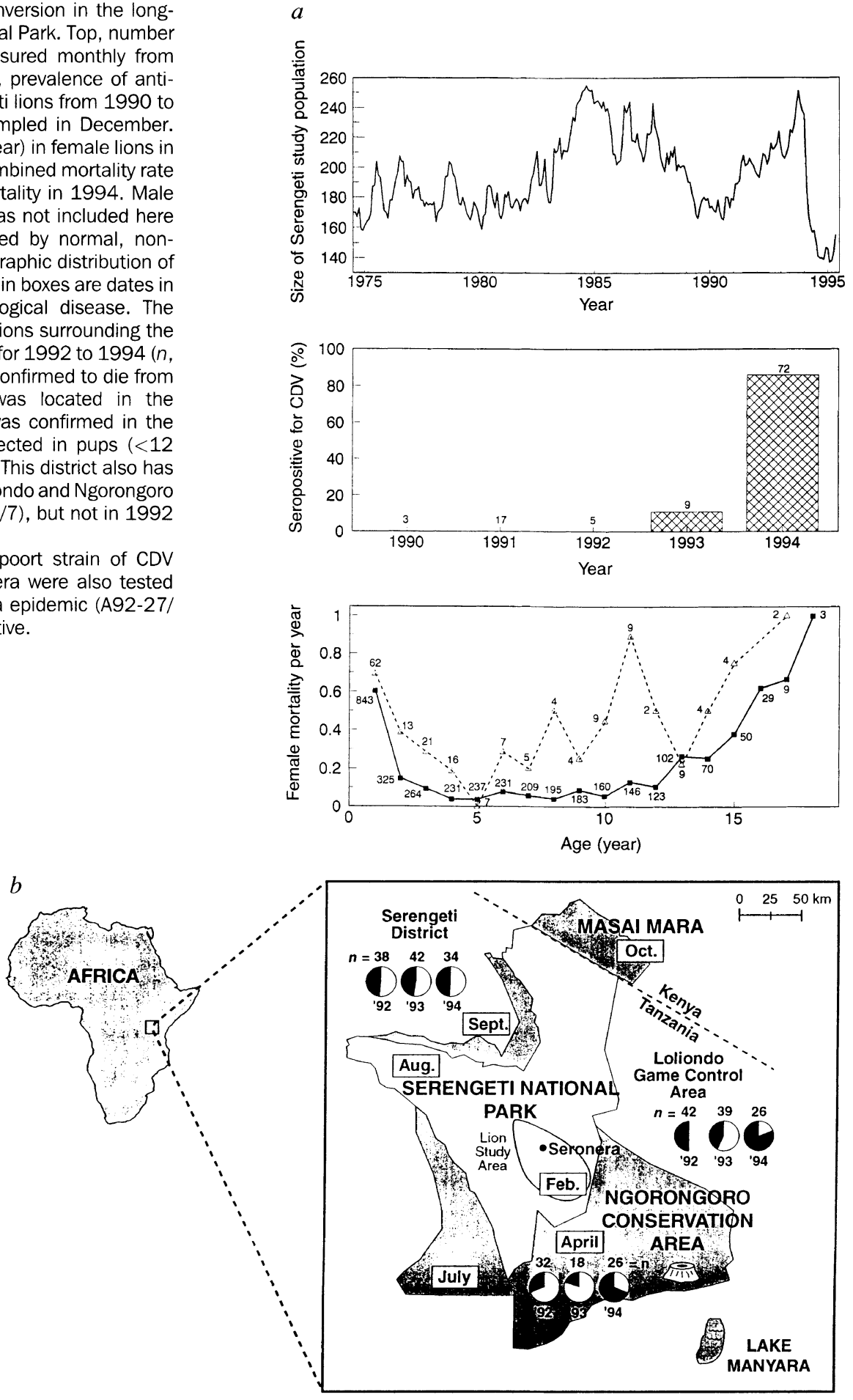

sampled in December of 1993 (Fig. 2a). Although this abrupt seroconversion indicates a recent exposure of these lions to CDV, analysis of sera from 77 lions in the region from 1984 to 1989 disclosed that 22 lions had antibodies to CDV (none of these previously tested lions was alive during the 1994 epidemic), indicating that lions in the Serengeti ecosystem had previously encountered CDV without an increase in disease-related mortality. To determine if the high lion mortality during the 1994 CDV epidemic was due to co-infection with another viral pathogen, we compared the prevalences of serum antibody titres to feline immunodeficiency virus (FeIV) ${ }^{15}$, feline parvovirus $(\mathrm{FePV})^{16}$, feline herpesvirus 1 (FeHV1), feline corona virus (FeCoV), and feline calici virus (FeCV) between lions with CDV disease $(6 / 10$ $\mathrm{FeIV}+$; 3/5 FePV+; 6/6 FeHV+; 2/6 FeCoV+; 1/6 FeCV+) and healthy CDV-seropositive lions (15/16 FeIV+; 11/12 FePV+; 13/ $13 \mathrm{FeHV}+$; 8/13 FeCoV+; 8/13 FeCV+). The discordance between CDV disease and antibodies to other viruses (Fisher's exact test: FeIV, $P>0.055$, FePV,$P>0.19$; FeCoV,$P>0.35$; $\mathrm{FeCV}, P>0.14$ ) fails to support a role for these viruses as necessary cofactors in CDV morbidity.

CDV infections in equatorial African wildlife usually occur as periodic epidemics because environmental factors limit viral persistence outside susceptible carnivore hosts, and these hosts usually succumb or rid themselves of virus ${ }^{9}$. CDV persists in dense populations of domestic dogs because pups provide a constant reserve of susceptible hosts. The source of CDV in the Serengeti epidemic was probably the domestic dogs of the local villages adjacent to the National Park. CDV seroprevalance increased in 

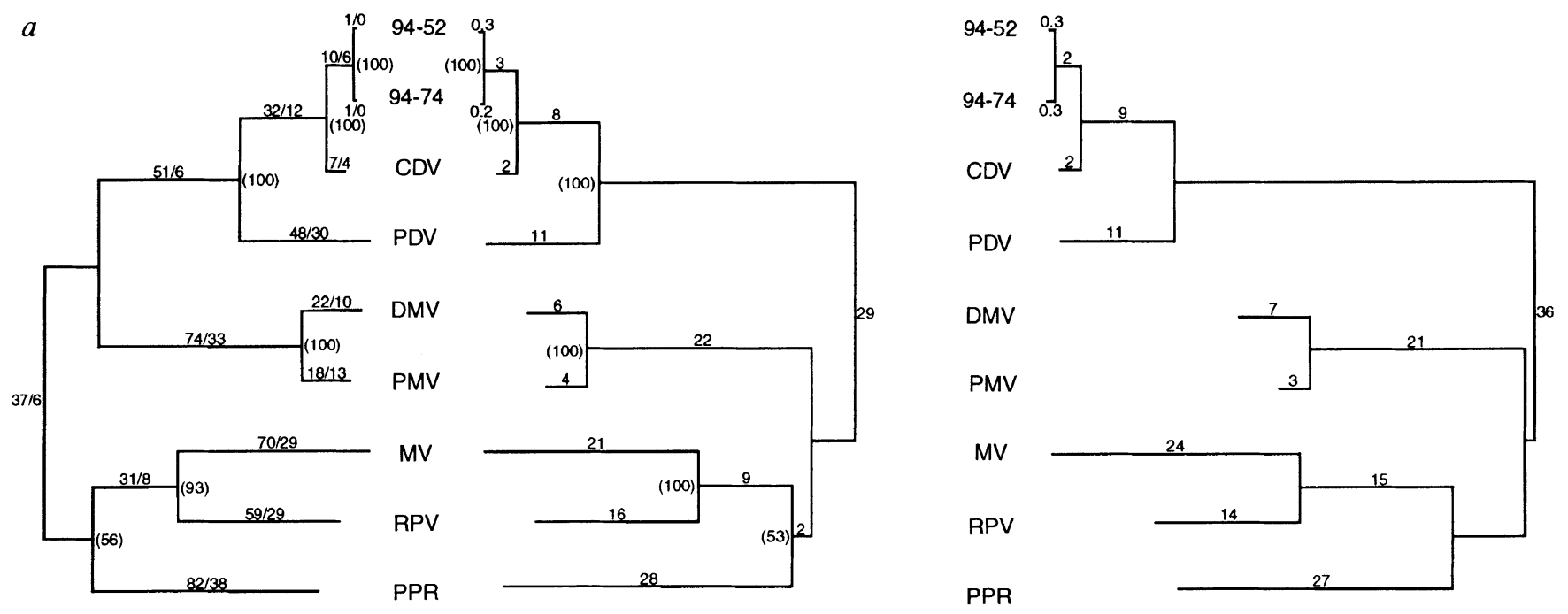

$b$

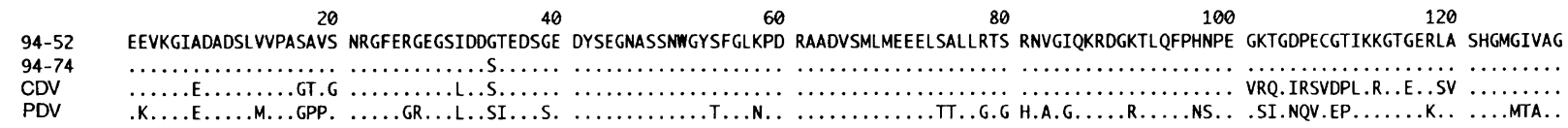

FIG. 3 Sequences and phylogenetic analysis of $389 \mathrm{bp}$ from lion morbillivirus $P$ genes. a, Viral RNA fragments of $429 \mathrm{bp}$ were amplified by RT-PCR from RNA of thymus and white blood cells isolated from two lions with neurological signs. Oligonucleotide primers were synthesized based on conserved regions of the CDV phosphoprotein $(P)$ gene as described previously ${ }^{18}$. PCR product fragments were cloned and sequenced, and the sequences aligned with P-gene sequences from other morbilliviruses. Derived P-gene sequences from two lions were 99\% identical. A phylogenetic analysis using cladistic, phenetic and maximum-likelihood methods revealed a close relationship between the lion morbillivirus and the Onderstepoort strain of CDV, the sequences having $95 \%$ nucleotide identity. PAUP maximum parsimony tree ${ }^{19}$ (left), with branch lengths equal to the number of nucleotide substitutions. Numbers shown on branches are

TABLE 1 Summary of CDV disease in Serengeti lions

\begin{tabular}{lc}
\multicolumn{1}{c}{ Criteria indicating CDV infection } & Number of affected lions \\
Neurological signs of CDV* & \\
$\quad$ Seizures & 12 \\
Myoclonus & 15 \\
Other neurological signs & 27 \\
Lion mortalities & \\
$\quad$ Carcasses recovered & 23 \\
$\quad$ Disappearances from observed & 31 \\
population of 250 lions & \\
CDV seroprevalence $\dagger$ & 71 of 83 \\
Lions with histopathological lesions & 18 of 19 \\
of CDV & 14 of 19 \\
Lions with viral inclusions in tissues & 14 of 19 \\
Lions with CDV nucleocapsid proteins \\
in tissues $\dagger$ \\
Lions with CDV isolated $\dagger$ \\
Lions with CDV RNA obtained by \\
RT-PCR $\ddagger$
\end{tabular}

* Number designates lions observed during 1994. Other neurological signs included ataxia, disorientation, profound depression or stupor, hyperaesthesia, and inappropriate behavioural responses.

$\dagger$ Immunohistochemistry and viral isolation were performed by methods published previously ${ }^{10,12}$. Buffy-coat isolation for viral cultures and molecular analyses were derived from $20 \mathrm{ml}$ blood taken from anaesthetized lions.

$\ddagger$ Methods are described in Fig. 3 legend. branch length and number of homoplasies, in that order. Tree length, 543; consistency index, 0.790. PHYLIP neighbour-joining tree ${ }^{20}$ (centre) generated using a transition/transversion ratio of 0.84 , with branch lengths equal to the percent of nucleotide differences. The number of bootstrap iterations (out of 100) which support the nodes are shown in parentheses. PHYLIP maximum-likelihood tree (right) generated with a transition/transversion ratio of 0.84 (in likelihood, $-2561.52597 ; 155$ trees examined). Branch lengths are the expected number of substitutions per site $\times 100$. 94-52-10 and 94-74 are sequences from Serengeti lions. CDV, canine distemper (Genbank X51869); PDV, phocine distemper virus (Genbank X75960); MV, measles virus (Genbank X16569); RPV, rinderpest virus (Genbank X68311); DMV, dolphin morbillivirus; PMV, porpoise morbillivirus; and PPR, peste des petits ruminants ${ }^{18} . b$, Translated amino-acid sequence of lion morbillivirus compared to CDV and PDV.

these dogs between 1991 and 1993, preceding the lion epidemic (Fig. 2b), and CDV encephalitis was confirmed by histopathology in a domestic dog in the Ngorongo Crater region in 1994. The precise route of CDV transmission to the lions is unclear, because direct dog-to-lion contact is unlikely for most of the Serengeti ecosystem. A more probable route is by spotted hyaenas, which range among human dwellings and travel long distances within the Park $^{17}$. Nomadic lions could also contribute to viral dissemination. High densities of these susceptible carnivores at kill sites would then provide an ideal environment for CDV amplification and transmission.

Most of the lion deaths in the Serengeti National Park occurred between January and September 1994, and mortality rates have subsequently returned to previous levels (Fig. 2a). Although this CDV epidemic claimed approximately $30 \%$ of the Serengeti and Mara lions, the impact on other carnivore species is unknown. Less dense populations of endangered species, such as cheetahs or wild dogs, are a clear cause for concern if exposed to a virulent pathogen such as this putative new biotype of CDV. The Serengeti is surrounded by approximately 30,000 domestic dogs, most of which are not vaccinated against canid pathogens (including CDV), representing a large reservoir for carnivore diseases. The CDV epidemic clearly emphasizes the need for continued disease surveillance to monitor infectious diseases in valuable wildlife resources, and for initiating vaccination programs for domestic animals in contact with wildlife.

\footnotetext{
Received 2 October; accepted 24 November 1994.
}

1. Moehlman, P. D. Recent Advances in the Study of Mammalian Behavior (eds Eisenberg, J. F. \& Kleiman, D. G.) 423-438 (American Society of Mammalogists, Lawrence, KA, 1983). 
2. Alexander, K. A. \& Appel, M. J. G. J. Wildl. Dis. 30, 481-485 (1994).

3. Macdonald, D. W. Nature 360, 633-634 (1992)

4. Packer, C. \& Pusey, A. E. Am. Nat. 145, 833-841 (1995).

5. Packer, C. et al. Reproductive Success (ed. Clutton-Brock, T. H.) 363-383 (Univ. Chicago Press, 1988)

6. Brown, E. W., Yuhki, N., Packer, C. \& O'Brien, S. J. J. Virol. 68, 5953-5968 (1994)

7. Gilbert, D. A., Packer, C., Pusey, A. E., Stephens, J. C. \& O'Brien, S. J. J. Hered. 82, 378-386 (1991).

8. Wildt, D. E. et al. Nature 329, 328-331 (1987)

9. Appel, M. J. G. Virus Infections in Carnivores (ed. Appel, M. J. G.) 133-160 (Elsevier, Amsterdam, 1987).

10. Appel, M. J. G. et al J. vet. diagnost. Invest. 6, 277-288 (1994)

11. Orvell, C., Sheshberadaran, H. \& Norrby, E. J. Virol. 66, 443-456 (1985)

12. Appel, M. J. G. \& Robson, D. S. Am. J. vet. Res. 34, 1459-1463 (1973).

13. Appel, M. J. G., Pearce-Kelling, S. \& Summers, B. A. J. vet. diagnost. Invest. 4, 258-263 (1992).

14. Montali, R. J., Bartz, C. R. \& Bush, M. Virus Infections of Carnivores (ed. Appel, M.) 437-443 (Elsevier, Amsterdam, 1991).

15. Brown, E. W. Miththapala, S. \& O'Brien, S. O. J. Zool. Wildl Med. 24, 357-364 (1993).

16. Carmichael, L. E., Jouber, J. C. \& Pollack, R. V. H. Am. J. vet. Res. 41, 784-791 (1980)

17. Hofer, H. \& East, M. L. Symp. zool. Soc. London 65, 347-366 (1993).

18. Barrett, T. et al. Virology 193, 1010-1012 (1993).

19. Swafford, D. L. Phylogenetic Analysis Using Parsimony (PAUP) (Illinois National History Survey, Champaign, IL, 1984)

20. Felsenstein, J. Phylogeny Inference Package (PHYLIP), Version 3.3 (University of Washington, Seattie, WA, 1995)

ACKNOWLEDGEMENTS. We thank M. Grob and M. Elgizoli for viral antigens, and M. East and $\mathrm{H}$. Hoeffer for providing two hyaena carcasses. This work was supported in part by the Messerli Foundation of Zurich, the University of Tennessee, Cornell University, Smithsonian NOAHS Center, the National Science Foundation, National Geographic, and British Airways.

\section{A common molecular basis for three inherited kidney stone diseases}

\author{
Sarah E. Lloyd*, Simon H. S. Pearce*, \\ Simon E. Fishert, Klaus Steinmeyer $\neq$, \\ Blanche Schwappach $\ddagger$, Steven J. Scheinman*§, \\ Brian Harding*, Alessandra Bolino\|, \\ Marcella Devoto\|, Paul Goodyerף, Susan P. A. \\ Rigden\#, Oliver Wrong ${ }^{i s}$, Thomas J. Jentsch $\ddagger$, \\ lan W. Craig $\dagger$ \& Rajesh V. Thakker* ${ }^{*} \dagger$
}

* MRC Molecular Endocrinology Group, Royal Postgraduate Medical School, Hammersmith Hospital, Du Cane Road, London W12 ONN, UK † Genetics Laboratory, University of Oxford, South Parks Road, Oxford OX1 3QU, UK

¥ Centre for Molecular Neurobiology Hamburg (ZMNH), Hamburg University, Martinistrasse 52, D-20246 Hamburg, Germany

$\S$ Department of Medicine, State University of New York Health Science Center, Syracuse, New York 13210, USA

| Laboratorio di Genetica Molecolare, Istituto G. Gaslini, Largo Gaslini 5 , 16148 Genova, Italy

I L'Hôpital de Montreal pour Enfants, 2300 rue Tupper, A717 Montreal, Quebec H3HIP3, Canada

\# Department of Paediatric Nephrology, Guy's Hospital,

St Thomas's Street, London SE1 9RT, UK

is Department of Nephrology, The Middlesex Hospital, Mortimer Street, London W1N 8AA, UK

KIDNEY stones (nephrolithiasis), which affect $12 \%$ of males and $5 \%$ of females in the western world, are familial in $45 \%$ of patients $^{1,2}$ and are most commonly associated with hypercalciuria ${ }^{1}$. Three disorders of hypercalciuric nephrolithiasis (Dent's disease ${ }^{3}, X$-linked recessive nephrolithiasis $(\mathrm{XRN})^{4}$, and $\mathrm{X}$-linked recessive hypophosphataemic rickets (XLRH) ${ }^{5}$ ) have been mapped to Xp11.22 (refs 5-7). A microdeletion ${ }^{6}$ in one Dent's disease kindred allowed the identification of a candidate gene, CLCN5 (refs 8,9) which encodes a putative renal chloride channel. Here we report the investigation of 11 kindreds with these renal tubular disorders for CLCN5 abnormalities; this identified three nonsense, four missense and two donor splice site

††To whom correspondence should be addressed. mutations, together with one intragenic deletion and one microdeletion encompassing the entire gene. Heterologous expression of wild-type CLCN5 in Xenopus oocytes yielded outwardly rectifying chloride currents, which were either abolished or markedly reduced by the mutations. The common aetiology for Dent's disease, XRN and XLRH indicates that $C L C N 5$ may be involved in other renal tubular disorders associated with kidney stones.

Analysis of CLCN5 reverse transcriptase-polymerase chain reaction (RT-PCR) products encompassing the entire 2238-bp coding sequence ${ }^{9}$ from probands of 11 kindreds with Dent's disease, XRN and XLRH, revealed different CLCN5 mutations (Table 1 and Fig. 1). Each mutation was confirmed and demonstrated to cosegregate with the disease by using genomic DNA together with the appropriate PCR primers and restriction enzymes, or by sequence-specific oligonucleotide (SSO) probe analysis (Table 1 and Fig. 2). In addition, the absence of these CLCN5 abnormalities in 110 alleles from 69 (28 males and 41 females) unrelated, normal individuals established that they were not common polymorphisms. CLCN5 belongs to a family of voltage-gated chloride-channel genes (CLCN1 to CLCN5 and $C L C N-K a$ and $K b$ ), which encode proteins (CLC-1 to CLC-5, CLC-Ka and CLC-Kb) that have about 12 transmembrane domains ${ }^{10}$. These chloride channels are important for the control of membrane excitability, transepithelial transport, and possibly regulation of cell volume ${ }^{10}$. However, mutations have been identified previously in only $C L C N 1$, which is expressed in muscle ${ }^{11}$ and is associated with myotonia congenita ${ }^{12-14}$. Thus, to assess further the functions of CLC-5 and its mutations, we performed heterologous expression studies in Xenopus oocytes. Expression of the human wild-type (WT) CLC-5 reproducibly yielded strongly outwardly rectifying, essentially time-independent currents (Fig. 3). Ion substitution experiments indicated that these were carried by anions, with a chloride $>$ iodide conductance sequence (Fig. $3 b, c$ ), as is the case with other chloride channels (CLC-0, CLC-1 and CLC-2) of this family ${ }^{12,15,16}$. However, these human CLC-5 currents, which were indistinguishable from those of rat CLC-5 (ref. 17), differed from the others in being strongly outwardly rectifying and in being observed only at potentials more positive than $+10 \mathrm{mV}$. Although positive potentials of even $+40 \mathrm{mV}$ have been observed in apical membranes of some actively transporting epithelia ${ }^{18}$, we are not aware of renal cells where these voltages would be reached in vivo. CLC channels are known to function as multimeric complexes, which are most likely to be tetramers $^{12}$, and it seems possible that CLC-5 forms heterooligomers with as yet unknown subunits in situ that may render the channels open at a more physiological voltage. Our functional expression of CLC-5 provides a valuable means of investigating this further and in assessing the functional effects of the CLC-5 mutations.

Expression of the four missense and three nonsense mutations (Table 1), and the in-frame deletion of the predicted transmembrane domain D2 (Fig. 1), abolished the CLC-5 currents or reduced them (S244L and S520P) to levels where they were difficult to distinguish from endogenous oocyte currents (Fig. $3 d, f)$. As a control, we also expressed more conservative changes at codons 244 and 520; S244T, S520T, S244A and S520A all elicited chloride currents that were similar to the WT (data not shown). Thus, the mutations found in the hypercalciuric nephrolithiasis pedigrees grossly and specifically affect CLC-5 function, thereby strongly suggesting a causal role in the disease. Dent's disease, which is characterized by low-molecular-weight proteinuria (LMWP), hypercalciuria, nephrocalcinosis, nephrolithiasis, rickets and eventual renal failure ${ }^{3,6}$, has phenotypic similarities to $\mathrm{XRN}^{4,7}$ and $\mathrm{XLRH}^{5}$ (Fig. 2). However, there are important differences, as rickets is absent in XRN, and nephrocalcinosis and moderate renal failure are more notable in XLRH. A correlation between these phenotypic differences, the different mutations (Table 1 and Fig. 1) and the resulting abnormal chloride currents (Fig. 3) could not be established. Thus, Dent's disease was found to be associated with the mutations W279X, 


\section{ERRATUM}

\section{doi:10.1038/nature08888}

\section{A canine distemper virus epidemic in Serengeti lions (Panthera leo)}

Melody E. Roelke-Parker, Linda Munson, Craig Packer, Richard Kock, Sarah Cleaveland, Margaret Carpenter, Stephen J. O'Brien, Andreas Pospischil, Regina Hofmann-Lehmann, Hans Lutz, George L. M. Mwamengele, M. N. Mgasa, G. A. Machange, Brian A. Summers \& Max J. G. Appel

Nature 379, 441-445 (1996)

In this Letter, the received and accepted dates for the manuscript were incorrectly listed as being in 1994, instead of 1995 . The correct dates are: received 2 October; accepted 24 November 1995. 